\title{
Probiotics and Gastrointestinal Health in Primary Care A National Survey in Spain
}

\author{
García Aguilar E and Leal Martínez-Bujanda J* \\ Medical Department, ITF Research Pharma, SLU, Alcobendas, Madrid, Spain \\ *Corresponding author: Leal Martínez-Bujanda Javier, Medical Department, ITF Research Pharma, SLU, Alcobendas, \\ Madrid, Spain
}

\section{ARTICLE INFO}

Received: March 13, 2020

Published: 幽 March 26, 2020

Citation: García Aguilar E, Leal Martínez-Bujanda J. Probiotics and Gastrointestinal Health in Primary Care. A National Survey in Spain. Biomed J Sci \& Tech Res 26(5)-2020. BJSTR. MS.ID.004402.

\begin{abstract}
Probiotics are an emerging tool to manage certain health conditions. Gastrointestinal disorders are common in general practice, and the primary care physician has a key role in the early detection and management of these problems. We have carried out a survey to determine the approach that is followed in patients with different gastrointestinal disturbances and the role that probiotics play in their treatment.
\end{abstract}

Keywords: Probiotic; Symbiotic; Clinical Practice Survey; Prodefen

Abbreviations: FAO: Food and Agriculture Organization; WHO: World Health Organization; CFU: Colony Forming Units; GRAS: Generally Recognized as Safe

\section{Introduction}

The gut microbiome seems to play a very important role in maintaining health including immune, metabolic and neurobehavioral traits. The birthing process exposes new-borns to a wide range of microorganisms that also contribute to the colonization of the gut microbiome. Despite exposure in utero, the majority of microbes that will colonize the infant gut are acquired postpartum. Gut microbiota composition transforms throughout early stages of human development and is influenced by the diet [1]. Globally the microbiome composition is noted to be different amongst different populations and cultures and it changes among life. It is documented that probiotics are capable of favourably modulate the intestinal ecosystem and be beneficial for those patients suffering from gastrointestinal diseases in which the gastrointestinal microbiota plays a key role. Indeed, probiotics have become an important tool for many health professionals in their daily routine. They have documented positive outcomes in different health benefits, although digestive health still remains the main focus of attention of these products.
Several species of the genera Bifidobacterium and Lacto bacillus are claimed to have a core benefit on healthy gut microbiota by creating a favorable gut environment.

According to the Food and Agriculture Organization/World Health Organization (FAO/WHO) Experts Committee, probiotics are defined as live microorganisms that, when administered in adequate amounts, confer a health benefit on the host [2]. In general, the microorganisms used as probiotics have a long history of safe usage and are considered GRAS (generally recognized as safe) [3]. Probiotics must contain alive microorganisms, which have to be resistant to acidic $\mathrm{pH}$ in the stomach as well as to bile to reach the small intestine and colon, where they exert their effects. Probiotics contain different microorganisms, most of which are similar bacteria to those present in our gut. Prior to this definition, only strain-specific probiotics were acknowledged to confer health benefits, and there is strong evidence supporting the hypothesis that the efficacy of probiotics is both strain-specific and disease-specific [4]; however, on the basis of a large number of well-designed clin- 
ical trials, it has been agreed that certain beneficial health effects of several strains of a number of well-studied microbial species can be ascribed to probiotics as a general class [5].

Several clinical studies and many medical reviews have revealed a positive effect of probiotics on gastrointestinal diseases such as diarrheas, inflammatory bowel disease, constipation, gastrointestinal disorders or irritable bowel syndrome among others. This effect appears to be linked to the role that probiotics have in maintaining an immunologic equilibrium in the gastrointestinal tract through direct interaction with immune cells [6]. The working mechanism of probiotics is based on the fact that they can interfere with pathogens, can improve barrier function, and have a role in immunomodulation and neurotransmitter production [7]. Diversity of the intestinal microbiome is emerging as a critical determinant of host health. There is evidence that different components of the microbiota have diverse and non-redundant contributions to host health [8]. In fact, dysbiosis defined as microbial imbalance, has been related with a wide variety of diseases and disorders.

Probiotic mixtures do not have an antagonistic effect on each other's survival when used in a multistrain product compared to a single-strain product [9]. Moreover, according to current scientific information, it is suggested that multistrain products might be more effective at reducing gastrointestinal infections $[10,11]$. Furthermore, it has been proposed that creating a mixture using species with different effects against different pathogens may have a broader spectrum of action that the one provided by a single strain $[12,13]$. A recent meta-analysis emphasizes the importance of the formulation. The authors suggest that combinations of strains of Lactobacillus and Bifidobacterium at a specific dose $\left(10^{9}-10^{10}\right.$ Colony Forming Units (CFU)) may achieve better results compared to those of single species or over-dosage products [11]. A symbiotic is a mixture of a probiotic and a prebiotic. Prebiotics are mostly fibers that are non-digestible food ingredients and that beneficially affect the health of the host by selectively stimulating the growth and/or activity of some genera of microorganisms in the colon, generally Lactobacilli and Bifidobacteria [14]. FAO/WHO defines prebiotics as a non-viable food component that confer health benefit(s) on the host associated with modulation of the microbiota [15].

Prodefen ${ }^{\circledR}$ is a symbiotic that contains a combination of prebiotics (fructooligosaccharides $990 \mathrm{mg}$ ) and seven probiotic strains (Lactobacillus rhamnosus, Lactobacillus casei, Streptococcus thermophilus, Bifidobacterium breve, Lactobacillus acidophilus, Bifidobacterium infantis, Bifidobacterium bulgaricus), per sachet. Prodefen ${ }^{\circledR}$ composition has proved to exert an additional benefit to the standard supportive therapy in the management of acute viral diarrhea in both hospitalized [16] and outpatient populations [17]. Prodefen ${ }^{\circledR}$ has also shown to significantly improve symptoms of infantile colic [18], to exert a positive effect on the eradication of $H$. pylori infection [19] and has demonstrated to improve symptoms of childhood constipation [20]. Recently, a new formulation for Prodefen ${ }^{\circledR}$ has been developed. Lactobacillus rhamnosus GG (LGG), $1 \times 10^{10} \mathrm{CFU}$, that is one of the most widely used probiotic strains [21] has been included in the formulation. LGG was the first strain belonging to the genus Lactobacillus to be patented in 1989 thanks to its ability to survive and to proliferate at gastric acid $\mathrm{pH}$ and in a medium containing bile, and to adhere to the enterocytes.

Furthermore, LGG is able to produce both a biofilm that can mechanically protect the mucosa and different soluble factors beneficial to the gut by enhancing intestinal crypt survival, diminishing apoptosis of the intestinal epithelium, and preserving cytoskeletal integrity. On the base of these functional properties that distinguish it from other probiotics, LGG is able to achieve significant results in the different situations characterized by microbiota dysbiosis [22]. Various health effects of LGG are well documented including the prevention and treatment of gastrointestinal infections and diarrhea, ameliorating irritable bowel disease symptomatology and stimulation of immune responses that promote vaccination or even prevent certain allergic symptoms [21,23-25]. LGG colonizes the gastrointestinal tract for 1-3 days in most individuals and up to 7 days after its administration [25]. This new formulation is branded as Prodefen Plus ${ }^{\circledR}$ and has been recently marketed in Spain with new evidence of a beneficial effect in preventing the antibiotic-associated diarrhea when administered as concomitant therapy in healthy adults treated with antibiotics [26].

Despite the widespread and accessible information that supports the benefits and usage of probiotics, health professionals may hesitate when recommending probiotics to patients due to the amount of confusing data and the wide offer of probiotics. In this regard, formulations that are supported by clinical evidence should be prioritized among others. Acute and chronic gastrointestinal disorders are common in general practice and the primary care physician plays an important and strategic role in the management of these digestive problems. As certain pathologies, such as gastroenteritis, involve frequent sick leave, it is also important to consider the subsequent socioeconomic costs and therefore the importance of selecting the right treatment. The purpose of this survey was to investigate the current knowledge and practice of primary health professionals regarding probiotics and the multistrain symbiotic Prodefen ${ }^{\circledR}$ in Spain.

\section{Material and Methods}

A self-completion 11-item on-line questionnaire was developed in order to describe the prevalence of patients affected with different gastrointestinal problems and the therapeutic approach regarding probiotics and these digestive disorders. The primary care 
specialists were contacted in Spain on July 2019. The questionnaire collected demographic data, characteristics and years of practice of the respondents, and information about their clinical management of some of the most commonly encountered gastrointestinal problems in primary care; such as flatulence, fullness and early satiety, abdominal bloating, abdominal pain and discomfort, heartburn, functional dyspepsia, diarrhea, constipation, bacterial overgrowth and Helicobacter Pylori infection. The recommendation of probiotics depending on the symptoms or gastrointestinal diagnosis and the reasons for considering or disregarding the use of probiotics were also collected.

\section{Results}

273 responses were received from primary care physicians Health professionals were drawn from all regions of Spain. Regarding the participants profile, $95 \%$ of the sample work for the public health service while only $5 \%$ run their practice exclusively in a private consultation. The median of years of practice was 20.7 with a median workload of 38.9 patients a day (778 patients per month). According to the data from the online questionnaire, the gastrointestinal problems evaluated are all prevalent in their primary care consultations, and $76 \%$ of the professionals treat them without referral. The most frequent gastrointestinal problems that were seen at the consultations (with an average of 6 to 15 patients a month) are abdominal pain and discomfort, flatulence, diarrhea, functional dyspepsia and constipation, while bacterial overgrowth and $\mathrm{H}$. Pylori infection are the least commonly reported. This sample of primary care practitioners reported to generally recommend probiotics to patients with these gastrointestinal disturbances. The percentages of recommendation according to each specific survey item is described in Figure 1.

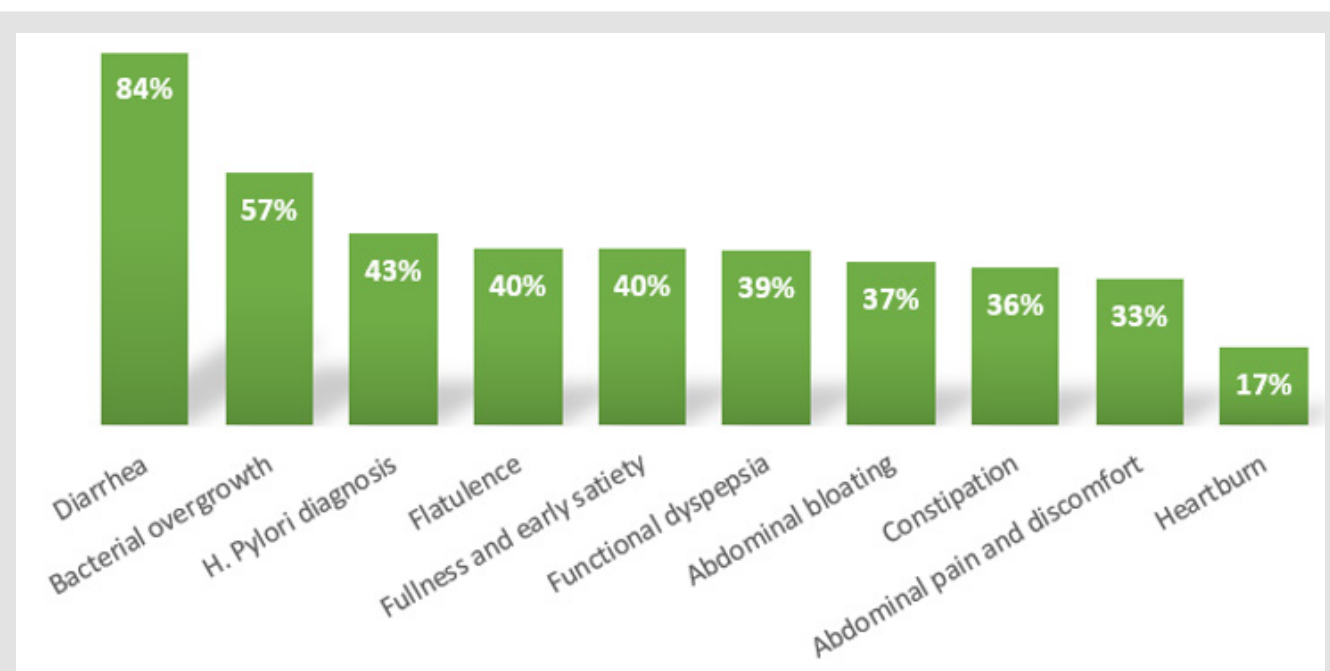

Figure 1: Percentage of primary care practitioners that recommend probiotics to their patients according to each gastrointestinal problem in Spain.

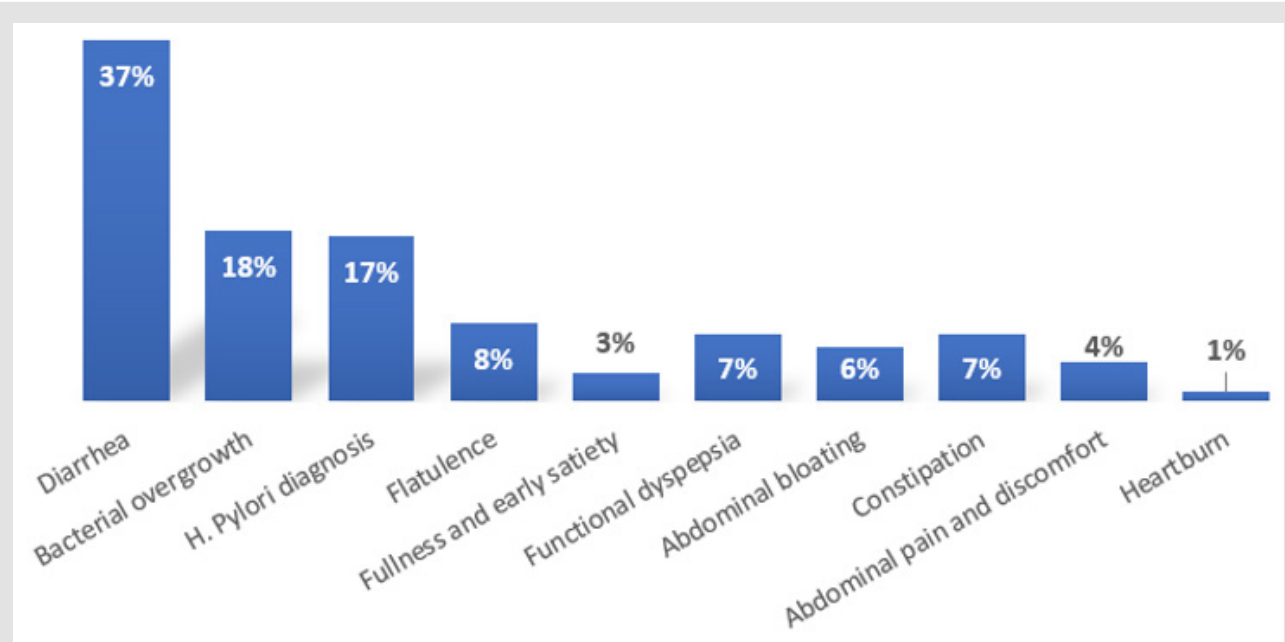

Figure 2: Percentage of primary care practitioners that always recommend a probiotic to their patients according to each gastrointestinal problem in Spain. 
In fact, there were some practitioners that would always recommend a probiotic in some specific gastrointestinal symptoms or diagnosis. These data are represented in Figure 2. Data on the drivers that influence the recommendation of a specific probiotic brand were also collected (Figure 3), being efficacy and safety the most relevant factors for the decision. Knowledge of the product, supporting clinical evidence, support from scientific societies, pharmaceutical presentation and the specific strains of the brand were also highly considered. Regarding the multistrain symbiotic brand Prodefen ${ }^{\circledR}$, primary care practitioners took into consideration, in addition to the previously mentioned drivers, the strain concentration and the innovation of the product as prominent factors versus other brands (Table 1 ). The vast majority of the respondents (85\%) considered that there is enough clinical evidence to advice the use of probiotics in gastrointestinal problems, and nearly half of them (41\%) would not be reluctant to recommend probiotics. $98 \%$ and $96 \%$ of the survey respondents would not hesitate to recommend a probiotic for safety or efficacy reasons, respectively. In fact, the most common issues that would negatively influence the recommendation of the physician were not related with the product itself, being the price the main barrier to advise the use of these products.

Table 1: Comparison of the mean punctuation of the factors that may impact on the primary care practitioner decision when recommending a probiotic brand and Prodefen ${ }^{\circledR}$ in Spain. From 0 (lowest relevance) to 10 (highest relevance).

\begin{tabular}{|c|c|c|}
\hline Drivers & Probiotics & Prodefen \\
\hline Safety & 9,2 & 9,2 \\
\hline Efficacy & 9,2 & 9 \\
\hline Knowledge of the product & 9 & 8,8 \\
\hline Brand specific clinical data & 8,8 & 8,8 \\
\hline Pharmaceutical presentation & 8,6 & 8,6 \\
\hline Scientific societies support & 8,8 & 8,6 \\
\hline Specific strains & 8,4 & 8,6 \\
\hline Multistrain & 8,4 & 8,4 \\
\hline Strain dose & 7,8 & 7,8 \\
\hline Cost & 7,6 & \multicolumn{2}{|c|}{7,6} \\
\hline Innovative & 6,8 & 7,4 \\
\hline Patients preferences & 7,2 & \\
\hline
\end{tabular}

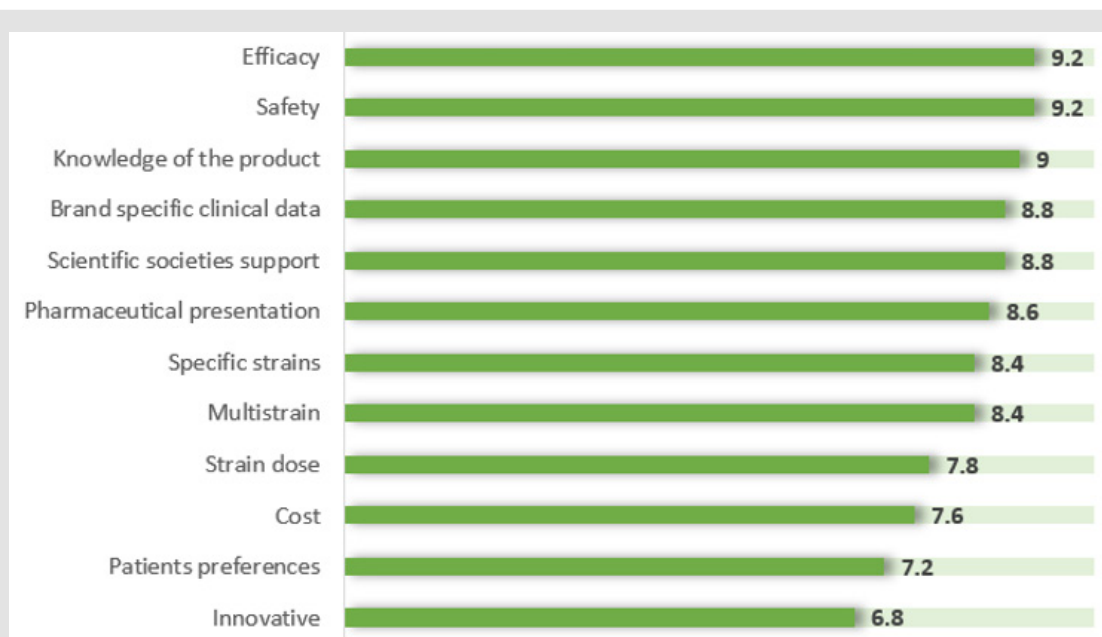

Figure 3: Mean punctuation of the factors that may impact on the primary care practitioner decision when recommending a probiotic brand in Spain. From 0 (lowest relevance) to 10 (highest relevance).

\section{Discussion}

One of the main objectives of our survey was to measure the role of the general practitioner in the management of certain digestive disorders. Digestive disorders are common in general practice and our results have confirmed that most of them are managed directly by the primary care physician. According to the results, over $80 \%$ of the patients referring flatulence, diarrhea or constipation and over $70 \%$ of the patients complaining about fullness and early satiety, abdominal bloating, abdominal pain and discomfort, heartburn, 
functional dyspepsia or diagnosed with Helicobacter Pylori infection are treated in primary care. These data support the importance of the primary care physician's role in the management of certain digestive pathologies. In Spain, primary care practitioners usually recommend probiotics for gastroenteritis and antibiotic-associated diarrhea. The benefits of probiotics in these medical conditions are well known. However, the persistent stream of publications about probiotics in gastrointestinal disorders allows clinicians to spread the use of probiotics in other settings beyond the above.

Although there is a large amount of scientific evidence supporting the benefits of probiotics, the wide range of probiotic products and certain strain-specific effects of probiotics have made it difficult for health professionals to make evidence-based decisions regarding probiotics. The outcomes of our survey tend to confirm the crucial role that the primary care physician has in the management of certain digestive disorders that are very prevalent and for which probiotics are recommended. According to our results, most primary clinicians are prone and confident to use probiotics in an important proportion of the patients affected with these problems, possibly influenced by their own clinical experience. In fact, while $37 \%$ of the primary care physicians would always recommend a probiotic to manage diarrhea, only $1 \%$ of the respondents would never recommend a probiotic for this condition. Being efficacy and safety the most important drivers for these recommendations, most clinicians considered probiotics both effective and safe. The brand (specific evidence and knowledge), presentation, composition and scientific support were also predisposing factors when recommending a probiotic.

Although the price was not one of the major drivers when recommending a probiotic, it was the main drawback for Spanish physicians to recommend a probiotic (49\% of the respondents considered it as a plausible handicap for prescription). Prodefen ${ }^{\circledR}$ brand is well known in Spain. According to our survey results, physicians seemed to be somewhat more inclined to recommend it when compared to other probiotics. The main differences in the drivers of recommendation were strain dose and the innovation of the product, which were better valued with Prodefen ${ }^{\circledR}$ compared to the general market, whilst the rest of the factors seemed very similar. These differences are possibly related to the fact that Prodefen ${ }^{\circledR}$ contains $10^{10} \mathrm{CFU}$, which fits within the range of recommendation for achieving a better probiotic efficacy [11] and to the knowledge of the product and the experience that clinicians have with the brand, being commercialized in Spain for almost 10 years and pursuing constant improvement (foresting both clinical development and innovation with new presentations/formulations).

\section{Conclusion}

Primary care practitioners are one of the main actors in the management of gastrointestinal conditions. Our survey provides interesting information about the approach and treatment of some of the most frequent digestive disorders they may face, focusing on the role that probiotics have as an option in their practice.

\section{References}

1. Gail A, Gresci (2015) The Gut Microbiome: What we do and don't know. Nutr Clin Pract Dec. 30(6): 734-746.

2. (2012) FAO/WHO, Editor. Guidelines for the Evaluation of Probiotics in Food.

3. Feride Karacaer, Hamed I, Özogul F, Glew RH, Özcengiz D, et al. (2017) The function of probiotics on the treatment of ventilator-associated pneumonia (VAP): Facts and gaps. Journal of Med Micr 66(9): 12751285.

4. McFarland LV, Evans CT, Goldstein EJC (2018) Strain-Specificity and Disease-Specificity of Probiotic Efficacy: A Systematic Review and MetaAnalysis. Font Med (Lausanne) 5: 124.

5. Sabina Fijan, Anita Frauwallner, László Varga, Tomaž Langerholc, Irena Rogelj, et al. (2019) Health Professionals' Knowledge of Probiotics: An International Survey. Int J Environ Res Public Health 16(17): 3128.

6. Ricci A, Tagliacarne SC, Valsecchi C (2015) Probiotics and inflammatory bowel diseases. J Biol Regul Homeost Agents 29(2 suppl 1): 96-113.

7. Sánchez B, Delgado S, Blanco Miguez A (2017) Probiotics gut microbiota and their influence on host health and disease. Mol Nutr Food Res 61(1).

8. Clarisse Petersen, June L Round (2014) Defining dysbiosis and its influence on host immunity and disease. Cell Microbiol 16(7): 10241033.

9. Forssten SD, Ouwehand AC (2017) Simulating colonic survival of probiotics in single-strain products compared to multi-strain products. Microbial Ecology in Health and Disease vol 28(1): 1378061.

10. Ford AC, Quigley EM, Lacy BE, Lembo AJ, Saito YA, et al. (2014) Efficacy of Prebiotics, Probiotics, and Synbiotics in Irritable Bowel Syndrome and Chronic Idiopathic Constipation: Systematic Review and Meta-analysis. Am J Gastroenterol 109: 1547-1561.

11. Ding Liang, Ning Longgui, Xu Guoqiang (2019) Efficacy of different probiotic protocols in irritable bowel syndrome. A network metaanalysis. Medicine 98(27): e16068.

12. Chapman CMC, Gibson GR, Rowland I (2012) In vitro evaluation of single- and multi-strain probiotics: Inter-species inhibition between probiotic strains and inhibition of pathogens. Anaerobe 18(4): 405-413.

13. Fijan S, Šulc IDD, Steyer A (2018) Study of the In Vitro Antagonistic Activity of Various Single-Strain and Multi-Strain Probiotics against Escherichia coli. Int J Environ Res Public Health 15(7): 1539.

14. DeVrese M, Schrezenmeir J (2008) Probiotics, prebiotics, and synbiotics. Adv Biochem Eng Biotechnol 111: 1-66.

15. (2007) Food and Agriculture Organization. FAO Technical Meeting on Prebiotics: Food Quality and Standards Service (AGNS), Food and Agriculture Organization of the United Nations (FAO); FAO Technical Meeting Report; FAO: Rome, Italy.

16. Allahverdi B, VAGHEI G, Foroughi S, Miri M, Delfan B, et al. (2010) A randomized clinical trial with probiotics in acute viral diarrhea in Iranian children. Proceeding of the $22^{\text {nd }}$ International Congress of Pediatrics.

17. Garcia Menor E, Garcia Marin F, Vecino Lopez R, Horcajo Martinez G, de Ibarrondo Guerrica-Echevarria MJ, (2016) et al. A Multicenter Prospective, Randomized Controlled Trial to Evaluate the Additional Benefit of a Multistrain Synbiotic (Prodefen(R)) in the Clinical Management of Acute Viral Diarrhea in Children. Glob Pediatr Health 3: $2333794 X 16679587$. 
18. Kianifar H, Ahanchian H, Grover Z, Jafari S, Noorbakhsh Z, et al. (2014) Synbiotic in the management of infantile colic: A randomized controlled trial. J Paediatr Child Health 50(10): 801-805.

19. Khodadad (2013) Probiotics for the treatment of Pediatric Helicobacter Pylori Infection: A Randomized Double-Blind Clinical Trial. Iran J Pediatr 23(1): 79-84

20. Sadeghzadeh, Rabieefar A, Khoshnevisasl P, Mousavinasab N, Eftekhari K, et al. (2014) The Effect of Probiotics on Childhood Constipation: A Randomized Controlled Double-Blind Clinical Trial. International Journal of Pediatrics, pp. 937212.

21. Segers ME, Lebeer S (2014) Towards a better understanding of Lactobacillus rhamnosus GG - host interactions. Microbial Cell Factories 13(Suppl 1): S7.

22. Lucio Capurso (2019) Thirty Years of Lactobacillus rhamnosus GG. J Clin Gastroenterol 53: S1-S41.

ISSN: 2574-1241

DOI: $10.26717 /$ BJSTR.2020.26.004402

Leal Martínez Bujanda. Biomed J Sci \& Tech Res

(C) This work is licensed under Creative

Submission Link: https://biomedres.us/submit-manuscript.php
23. Szajewska H, Kolodziej M (2015) Systematic review with meta-analysis: Lactobacillus rhamnosus GG in the prevention of antibiotic-associated diarrhoea in children and adults. Aliment Pharmacol Ther 42(10): 1149 1157.

24. Pedersen en, Nynne Nyboe Andersen, Zsuzsanna Végh, Lisbeth Jensen, Dorit Vedel Ankersen, et al. (2014) Ehealth: Low FODMAP diet vs Lactobacillus rhamnosus GG in irritable bowel syndrome. World J Gastroenterol 20(43): 16215-16226.

25. Gorbach SL (2000) Probiotics and gastrointestinal health. Am J Gastroenterol 95(1 Suppl): S2-S4

26. Serena AR (2019) Efficacy of a 7-strain synbiotic mixture in combination with oral antibiotic treatment in preventing antibiotic-associated diarrhea (AAD). PRODEGGIO Study Presented in the World Congress of Gastroenterology, Istanbul, Turkey, p. 21-24.

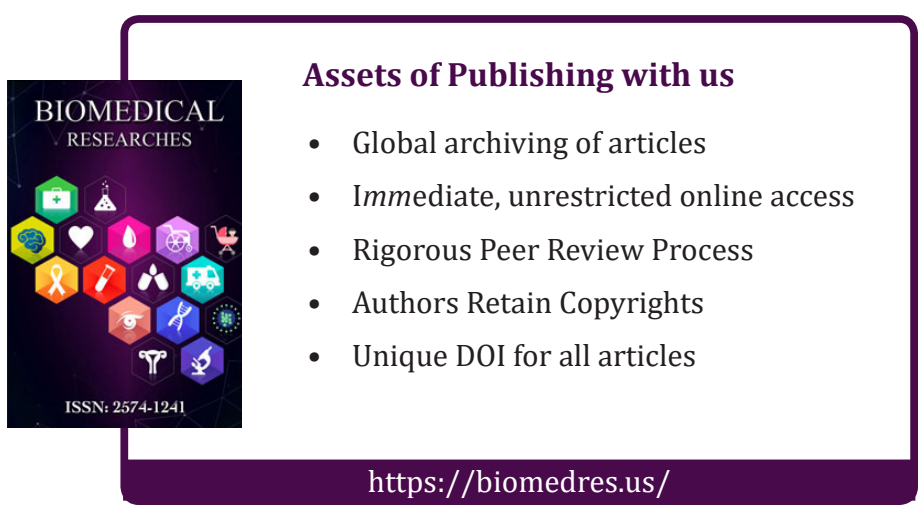

